\title{
Oscillation for second order nonlinear delay differential equations with impulses
}

\author{
THIAGARAJAN REVATHI \\ Department of mathematics, Queen Mary's College Chennai-600004,TamilNadu
} \begin{abstract}
equations with impulses of the form

$$
\begin{aligned}
& {\left[r(t) x^{\prime}(t)\right]^{\prime}+p(t) x^{\prime}(t)+Q(t, x(t-\delta))=0, t \geq t_{0}, t \neq t_{k}, k=1,2, \ldots n} \\
& x\left(t_{k}^{+}\right)=g_{k}\left(x\left(t_{k}\right)\right), x^{\prime}\left(t_{k}^{+}\right)=h_{k}\left(x^{\prime}\left(t_{k}^{+}\right)\right)
\end{aligned}
$$
\end{abstract}

Abstract: In this paper, we investigate the oscillation of second order nonlinear delay differential

Key words :Oscillation; delay; second-order;impulses

Mathematics Subject classification 34A37

\section{Introduction}

The theory of impulsive differential equations is now being recognized to be not only richer than the corresponding theory of differential equations without impulses, but also represents a more natural frame work for the mathematical modeling of many real work phenomena[1].There are many papers devoted for the oscillation criteria of second-order differential equations without impulses[2-3], with impulse[4-7] .In[8],the authors obtained the asymptotic behavior for the equations

$$
\begin{aligned}
& {\left[r(t) x^{\prime}(t)\right]^{\prime}+p(t) x^{\prime}(t)+Q(t, x(t-\delta))=0, t \geq t_{0}, t \neq t_{k}, k=1,2, \ldots n} \\
& x\left(t_{k}^{+}\right)=g_{k}\left(x\left(t_{k}\right)\right), x^{\prime}\left(t_{k}^{+}\right)=h_{k}\left(x^{\prime}\left(t_{k}^{+}\right)\right)
\end{aligned}
$$

In this paper we obtain the oscillation of second order nonlinear delay differential equations with impulses of the form

$$
\begin{aligned}
& {\left[r(t) x^{\prime}(t)\right]^{\prime}+p(t) x^{\prime}(t)+Q(t, x(t-\delta))=0, t \geq t_{0}, t \neq t_{k}, k=1,2, \ldots n} \\
& x\left(t_{k}^{+}\right)=g_{k}\left(x\left(t_{k}\right)\right), x^{\prime}\left(t_{k}^{+}\right)=h_{k}\left(x^{\prime}\left(t_{k}^{+}\right)\right) \\
& x(t)=\phi(t), x\left(t_{0}^{+}\right)=x_{0}, \quad x\left(t_{0}^{+}\right)=x_{0}^{\prime}, t \in\left[t_{0}-\delta, t_{0}\right]
\end{aligned}
$$

where for every $t_{0} \geq 0, \phi \in P C_{t 0}=\left\{\phi:\left[t_{0}-\delta, t_{0}\right] \rightarrow+\infty / \phi\right\}$

A function $x(t)$ is said to be a solution of (1) and (2) satifying the initial value condition (3) if

(i) $x:\left[t_{0}-\delta, \infty\right] \rightarrow \square$ satisfies(1.3) for $t_{0}-\delta \leq t \leq t_{0} ; t \geq t_{0}, x\left(t_{k}^{+}\right), x\left(t_{k}^{+}\right), x\left(t_{k}^{-}\right)$and $x\left(t_{k}^{-}\right)$

(ii) $x(t)$ and $x^{\prime}(t)$ are continuously differentiable for $\mathrm{t}>t_{0}, t \neq t_{k} t \neq t_{k}+\delta$ and satisfies

(1);

(iii) for $t \geq t_{0,} x\left(t_{k}^{+}\right), x^{\prime}\left(t_{k}^{+}\right), x\left(t_{k}^{-}\right)$and $x^{\prime}\left(t_{k}^{-}\right)$exist with $x\left(t_{k}^{+}\right)=x\left(t_{k}\right), x^{\prime}\left(t_{k}^{+}\right)=x^{\prime}\left(t_{k}\right)$ and satisfy (2)

As is customary, a solution of (1) and (2) is said to be non oscillatory, if it is eventually positive or eventually negative. Otherwise it will be called oscillatory.

Here, we always assume

(H1) $Q(t, x)$ is continuous

in $\left[t_{0}, \infty\right), x Q(t, x)>0(x \neq 0)$ and $Q(t, x) / f(x) \geq q(t) \quad(x \neq 0)$ where $q(t)$ is continuous

in $\left[t_{0}, \infty\right)$ and $q(t) \geq 0, x f(x) .0, f^{\prime}(x) \geq k>0, r(t)>0$. 
(H2) $p(t), g_{k}(x), h_{k}(x)$ are continuous in $\mathrm{R}$ and there exist positive numbers $a_{k}, a_{k}^{*}, b_{k}, b_{k}^{*}$ such that $a_{k}^{*} \leq \frac{g_{k}(x)}{x} \leq a_{k}, b_{k}^{*} \leq \frac{h_{k}(x)}{x} \leq b_{k}$.

Lemma 1 Let $\mathrm{x}(\mathrm{t})$ be a solution of (1) and (2).Suppose that there exists some $T \geq t_{0}$ suchthat $x(t)>0, t \geq T$. If (H1) and (H2) are satisfied and

$$
\begin{aligned}
& \sum_{m=1}^{n-1} \prod_{k=m}^{n-1} \prod_{l=0}^{m-1} a_{k} b_{1}^{*} \int_{t_{m-1}}^{t_{m}} \exp \left[\int_{t_{0}}^{u} \frac{r^{\prime}(s)+p(s)}{r(s)} d s\right] d u \\
& +\prod_{k=0}^{n-1} b_{1}^{*} \int_{t_{n-1}}^{t_{n}}\left[-\int_{t_{0}}^{u} \frac{r^{\prime}(s)+p(s)}{r(s)} d s\right] d u=+\infty
\end{aligned}
$$

holds, then $x^{\prime}\left(t_{j}\right)>0$ and $x^{\prime}(t)>0$ for $t \in\left(t_{k}, t_{k+1}\right]$, where $t_{k} \geq T, k=1,2, \ldots n$.

Proof. At first,we prove that $x^{\prime}\left(t_{k}\right)>0$ for any $t_{k} \geq T$. If it is not true , then there exist some $\mathrm{j}$ such that $t_{j} \geq T, x^{\prime}\left(t_{j}^{+}\right)<0$. Then $x^{\prime}\left(t_{j}^{+}\right)=h_{j}\left(x^{\prime}\left(t_{j}\right)\right) \leq b_{j}^{*} x^{\prime}\left(t_{j}\right)$.By (1),we have

$x^{\prime \prime}(t)+\frac{r^{\prime}(t)+p(t)}{r(t)} x^{\prime}(t)+\frac{Q(t, x)}{r(t)}=0$,

i.e.,

$\left[x^{\prime}(t) \exp \left(\int_{t_{j}}^{t} \frac{r^{\prime}(s)+p(s)}{r(s)} d s\right)\right]^{\prime}=-\frac{Q(t, x)}{r(t)} \exp \left(\int_{t_{j}}^{t} \frac{r^{\prime}(s)+p(s)}{r(s)} d s\right) \leq 0$, $t \in\left(t_{j}, t_{j+1}\right]$

Hence $\quad x^{\prime}(t) \exp \left(\int_{t_{j}}^{t} \frac{r^{\prime}(s)+p(s)}{r(s)} d s\right)$

is decreasing in $\left(t_{j}, t_{j+1}\right]$,

$$
\begin{aligned}
& x^{\prime}\left(t_{j+1}\right) \exp \left(\int_{t_{j}}^{t_{j+1}} \frac{r^{\prime}(s)+p(s)}{r(s)} d s\right) \leq x^{\prime}\left(t_{j}^{+}\right) \leq b_{j}^{*} x^{\prime}\left(t_{j}\right), \\
& x^{\prime}\left(t_{j+1}\right) \leq b_{j}^{*} x^{\prime}\left(t_{j}\right) \exp \left(-\int_{t_{j}}^{t_{j+1}} \frac{r^{\prime}(s)+p(s)}{r(s)} d s\right) .
\end{aligned}
$$

For $t \in\left(t_{j+1}, t_{j+2}\right]$, we have

$$
x^{\prime}\left(t_{j+2}\right) \leq b_{j+1}^{*} b_{j}^{*} x^{\prime}\left(t_{j}\right) \exp \left(-\int_{t_{j+1}}^{t_{j+2}} \frac{r^{\prime}(s)+p(s)}{r(s)} d s\right) \text {. }
$$

It is easy to show that for any natural number $n \geq 2$

$$
x^{\prime}\left(t_{j+n}\right) \leq \prod_{j=0}^{n-1} b_{j+k}^{*} x^{\prime}\left(t_{j}\right) \exp \left(-\int_{t_{j+1}}^{t_{j+n}} \frac{r^{\prime}(s)+p(s)}{r(s)} d s\right) \text {. }
$$

Since 
$x^{\prime}(t) \exp \left(\int_{t_{j}}^{t} \frac{r^{\prime}(s)+p(s)}{r(s)} d s\right)$

is decreasing in $\left(t_{j}, t_{j+1}\right]$,hence,

$x^{\prime}(t) \leq x^{\prime}\left(t_{j}^{+}\right) \exp \left(-\int_{t_{j}}^{t} \frac{r^{\prime}(s)+p(s)}{r(s)} d s\right), t \in\left(t_{j}, t_{j+1}\right]$.

Integrating the above inequality from $s$ to $t$, we have

$$
x(t) \leq x(s)+x^{\prime}\left(t_{j}^{+}\right) \int_{s}^{t} \exp \left(-\int_{t_{j}}^{u} \frac{r^{\prime}(s)+p(s)}{r(s)} d s\right) d u, t_{j}<s \leq t \leq t_{j+1} .
$$

Let $t \rightarrow t_{j+1}, s \rightarrow t_{j}^{+}$, we get

$$
\begin{aligned}
& x\left(t_{j+1}\right) \leq x\left(t_{j}^{+}\right)+x^{\prime}\left(t_{j}^{+}\right) \int_{t_{j}}^{t_{j+1}} \exp \left(-\int_{t_{j}}^{u} \frac{r^{\prime}(s)+p(s)}{r(s)} d s\right) d u, \\
& \leq a_{j} x\left(t_{j}\right)+b_{j}^{*} x^{\prime}\left(t_{j}\right) \int_{t_{j}}^{t_{j+1}} \exp \left(-\int_{t_{j}}^{u} \frac{r^{\prime}(s)+p(s)}{r(s)} d s\right) d u, \\
& x\left(t_{j+2}\right) \leq a_{j+1} a_{j} x\left(t_{j}\right)+a_{j+1} b_{j}^{*} x^{\prime}\left(t_{j}\right) \int_{t_{j}}^{t_{j+1}} \exp \left(-\int_{t_{j}}^{u} \frac{r^{\prime}(s)+p(s)}{r(s)} d s\right) d u+b_{j+1}^{*} b_{j}^{*} x^{\prime}\left(t_{j}\right) \int_{t_{j}}^{t_{j+1}} \exp \left(-\int_{t_{j}}^{u} \frac{r^{\prime}(s)+p(s)}{r(s)} d s\right) d u
\end{aligned}
$$

By induction for any natural number $\mathrm{n}$, we have

$$
x\left(t_{j+n}\right) \leq \prod_{k=0}^{n-1} a_{j+k} x\left(t_{j}\right)+x^{\prime}\left(t_{j}\right) \sum_{m=1}^{n-1} \prod_{k=m}^{n-1} \prod_{l=0}^{m-1} a_{j+k} b_{j+l}^{*} \int_{t_{j+m-1}}^{t_{j+m}} \exp \left(-\int_{t_{j}}^{u} \frac{r^{\prime}(s)+p(s)}{r(s)} d s\right) d u+\prod_{k=0}^{n-1} b_{j+k}^{*} \int_{t_{j+n-1}}^{t_{j+n}} \exp \left(-\int_{t_{0}}^{u} \frac{r^{\prime}(s)+p(s)}{r(s)} d s\right) d u
$$

since , $x(t)>0, x^{\prime}\left(t_{j}\right) \geq 0\left(t_{j} \geq T\right) \quad, \quad$ the above inequality is contrary to condition

(4).Therefore $x^{\prime}\left(t_{k}\right) \geq 0\left(t_{k} \geq T\right)$.Because

$$
\begin{aligned}
& x^{\prime}(t) \exp \left(\int_{t_{j}}^{t} \frac{r^{\prime}(s)+p(s)}{r(s)} d s\right) \text { is decreasing in }\left(t_{j}, t_{j+1}\right], \\
& x^{\prime}(t) \exp \left(\int_{t_{j}}^{t} \frac{r^{\prime}(s)+p(s)}{r(s)} d s\right) \geq x^{\prime}\left(t_{j+1}\right) \exp \left(\int_{t_{j}}^{t_{j+1}} \frac{r^{\prime}(s)+p(s)}{r(s)} d s\right) \geq 0
\end{aligned}
$$

Hence $x^{\prime}(t) \geq 0, t \in\left(t_{k}, t_{k+1}\right]$.

The proof of Lemma 1 is complete. 


\section{Theorem 1:}

If conditions (1) - (3) hold, then $a_{k}^{*}<1$ and there exist $G(t) \geq 0(\leq 0), F(t) \geq 0$ such that the following equa $\sum_{m=1}^{n} \prod_{j=m}^{n} b_{j}\left(\psi-\frac{k a(t) r(t)}{4} F^{2}(t)\right) \exp \left[\int_{t_{n}}^{t} \frac{p(s)}{r(s)}+k F(s) d s\right] d t=+\infty$,

where

$$
\begin{aligned}
& \psi(t)-k^{-1} a(t)\left[k q(t)-p(t) G(t)-[r(t) G(t)]^{\prime}+r(t) G^{2}(t)\right], \\
& a(t)=\exp \left[-2 \int_{0}^{t} G(u) d u\right] .
\end{aligned}
$$

Proof.Let $\mathrm{x}(\mathrm{t})$ be a nonoscillatory solution of the differential equation (1) and let $T_{0} \geq t_{0}$ be such that $x(t) \neq 0$ for all $t \geq t_{0}$. Without loss of generality, we assume that $x(t)>0$ for all $t \geq T_{0}$. In the following, we $v(t)=a(t) r(t)\left[\frac{x^{\prime}(t)}{f(x(t-\delta))}+\frac{G(t)}{k}\right]$.

Differentiating the equality and making use of (1) and (H1) we get

$$
\begin{aligned}
v^{\prime}(t) & \leq-\psi(t)-\frac{p(t) v(t)}{r(t)}-\frac{k v^{2}(t)}{a(t) r(t)}+\frac{k a(t) r(t)}{4} F^{2}(t)-\frac{k a(t) r(t)}{4} F^{2}(t) \\
& \leq-\left(\psi(t)-\frac{k a(t) r(t)}{4} F^{2}(t)\right)-\left(k F(t)+\frac{p(t)}{r(t)}\right) v(t) .
\end{aligned}
$$

For all $t \geq T_{0}, t \neq t_{k}$, with $\psi(t)$ defined as above, by (4)

$$
\begin{aligned}
v(t) & \exp \left[\int_{t_{j}}^{t} \frac{p(s)}{r(s)}+k F(s) d s\right]^{\prime} \\
& \leq-\left(\psi(t)-\frac{k a(t) r(t)}{4} F^{2}(t)\right) \exp \left[\int_{t_{j}}^{t} \frac{p(s)}{r(s)}+k F(s) d s\right] .
\end{aligned}
$$

Integrating from sto $s_{1}$

$$
\begin{aligned}
v\left(s_{1}\right) & \leq v(s) \exp \left[\int_{s_{1}}^{s} \frac{p(s)}{r(s)}+k F(s) d s\right]-\int_{s}^{s_{1}}\left[\psi(t)-\frac{k a(t) r(t)}{4} F^{2}(t)\right] \\
& \times \exp \left[\int_{t_{j}}^{t} \frac{p(s)}{r(s)}+k F(s) d s\right] \\
v\left(t_{k}^{+}\right)= & a\left(t_{k}\right) r\left(t_{k}\right)\left[\frac{x^{\prime}\left(t_{k}^{+}\right)}{f\left(x_{k}\right)}+\frac{G\left(t_{k}\right)}{k}\right] .
\end{aligned}
$$

In (8) let $s_{1}=t_{1}, s_{0}=t_{0}^{+}$. Then 


$$
\begin{aligned}
v\left(t_{1}^{+}\right) & \leq b_{1} \leq b_{1} v\left(t_{0}^{+}\right) \exp \left[\int_{t_{1}}^{t_{0}} \frac{p(s)}{r(s)}+k F(s) d s\right] \\
& -\int_{t_{0}}^{t_{1}}\left[\psi(t)-\frac{k a(t) r(t)}{4} F^{2}(t)\right] \exp \left[\int_{t_{1}}^{t_{0}} \frac{p(s)}{r(s)}+k F(s) d s\right] .
\end{aligned}
$$

By induction, for any natural number $\mathrm{n}$, we have

$$
\begin{aligned}
& v\left(t_{n}^{+}\right) \leq \prod_{k=1}^{n} b_{k} v\left(t_{0}^{+}\right) \exp \left[\int_{t_{n}}^{t_{0}} \frac{p(s)}{r(s)}+k F(s) d s\right] \\
& \times \sum_{m=1}^{n} \prod_{j=m}^{n} b_{j}\left[\psi(t)-\frac{k a(t) r(t) F^{2}(t)}{4}(t)\right] \exp \left[\int_{t_{1}}^{t_{0}} \frac{p(s)}{r(s)}+k F(s) d s\right] .
\end{aligned}
$$

By the condition of theorem 1 and $v\left(t_{0}^{+}\right) \geq 0$, the above inequality is impossible. The proof of theorem is completed.

\section{Theorem 2.}

Assume that the condition of lemma 1 hold and $f(a b) \geq f(a) f(b)$ for any $a b>0, f^{\left(a_{k}^{*}\right)} / b_{k} \leq 1$ for $k \geq 1$.If there exist $F(t), G(t) \geq 0$ such that

$$
\sum_{m=1}^{n} \prod_{j=m}^{n} \frac{b_{j}}{f\left(a_{j} *\right)_{t_{m-1}}^{t_{m}}} \int_{t_{n}} \psi(t)-\frac{k a(t) r(t)}{4} F^{2}(t) \exp \left[\int_{t_{n}}^{t} \frac{p(s)}{r(s)}+k F(s) d s\right] d t=+\infty,
$$

then every solution of 1 is oscillatory

Proof. With loss of generality, we assume that $\mathrm{x}(\mathrm{t})>0$.By Lemma $(1), x^{\prime}(t) \geq 0$.

Let

$$
v(t)=a(t) r(t)\left[\frac{x^{\prime}(t)}{f(x)}+\frac{G(t)}{k}\right]
$$

Then $v(t) \geq 0, v\left(t_{k}^{+}\right) \geq 0$. Relation (1) yields

$$
v\left(t_{k}^{+}\right) \leq \frac{b_{k}}{f\left(a_{k}^{*}\right)} v\left(t_{k}\right) .
$$

Following the similar way to the proof of theorem 1, the proof is omitted . 


\section{Theorem 3.}

Suppose the conditions of Lemma1 hold and there exists a positive integer $k_{0}$ such that $a_{k}^{*}>1$ for $k \geq k_{0}$.If

$$
\begin{aligned}
& \int_{\in}^{+\infty} \frac{d u}{f(u)}<+\infty\left(\int_{\infty}^{-\epsilon} \frac{d u}{f(u)}>-\infty\right), \\
& \sum_{k=0}^{\infty} \int_{t_{k}}^{t_{k+1}} \sum_{j=1}^{n-1} \sum_{i=1}^{j} \frac{1}{b_{k+i}} \int_{t_{k+j}}^{t_{k+j+1}} \frac{q(s)}{r(s)} \exp \left[\int_{t_{k+j}}^{t} \frac{r^{\prime}(u)+p(u)}{r(u)} d u\right] d s \\
& \quad \times \exp \left[-\int_{t_{k+1}}^{t} \frac{r^{\prime}(u)+p(u)}{r(u)} d u\right]+\exp \left[-\int_{t_{k+1}}^{t} \frac{r^{\prime}(u)+p(u)}{r(u)} d u\right] \\
& \quad \times \exp \left[\int_{t_{k+1}}^{s} \frac{r^{\prime}(u)+p(u)}{r(u)} d u\right] d s=+\infty \\
& \quad \text { hold, then every solution of (1) is oscillatory }
\end{aligned}
$$

Proof. Without loss of generality, we assume that $\mathrm{x}(\mathrm{t})>0$ for all $t \geq T_{0}, k_{0}=1$.

By (2) we know

$$
\begin{aligned}
& x^{\prime}\left(t_{0}^{+}\right) \geq x^{\prime}\left(t_{1}\right) \exp \left(\int_{t_{0}}^{t_{1}} \frac{r^{\prime}(s)+p(s)}{r(s)} d s\right)+\int_{t_{0}}^{t_{1}} \frac{Q(t, x)}{r(t)} \\
& \times \exp \left(\int_{t_{0}}^{t_{1}} \frac{r^{\prime}(s)+p(s)}{r(s)} d s\right) d t, \\
& x^{\prime}\left(t_{1}^{+}\right) \geq \frac{x^{\prime}\left(t_{2}^{+}\right)}{b 2} \exp \left(\int_{t_{1}}^{t_{2}} \frac{r^{\prime}(s)+p(s)}{r(s)} d s\right)+\int_{t_{1}}^{t_{2}} \frac{Q(t, x)}{r(t)} \\
& \times \exp \left(\int_{t_{1}}^{t} \frac{r^{\prime}(s)+p(s)}{r(s)} d s\right) d t .
\end{aligned}
$$

By induction for any natural number $\mathrm{k}$, we have

$$
\begin{aligned}
& x^{\prime}\left(t_{k}^{+}\right) \geq \frac{x^{\prime}\left(t_{k+1}^{+}\right)}{b_{k+1}} \exp \left(\int_{t_{k}}^{t_{k+1}} \frac{r^{\prime}(s)+p(s)}{r(s)} d s\right)+\int_{t_{k}}^{t_{k+1}} \frac{Q(t, x)}{r(t)} \\
& \times \exp \left(\int_{t_{k}}^{t} \frac{r^{\prime}(s)+p(s)}{r(s)} d s\right) d t .
\end{aligned}
$$


From the above and (2) noting $x^{\prime}\left(t_{k}^{+}\right) \geq 0$, we get, for $t \in\left(t_{k}, t_{k+1}\right]$,

$$
\begin{aligned}
& \int_{x_{0}}^{+\infty} \frac{d u}{f(u)} \geq \sum_{k=0}^{\infty} \int_{t_{k}}^{t_{k+1}} \sum_{j=1}^{n-1} \sum_{i=1}^{j} \frac{1}{b_{k+i}} \int_{t_{k+j}}^{t_{k+j+1}} \frac{q(s)}{r(s)} \exp \left[\int_{t_{k+j}}^{t} \frac{r^{\prime}(u)+p(u)}{r(u)} d u\right] d s \\
& \quad \times \exp \left[-\int_{t_{k+1}}^{t} \frac{r^{\prime}(u)+p(u)}{r(u)} d u\right]+\exp \left[-\int_{t_{k+j}}^{t} \frac{r^{\prime}(u)+p(u)}{r(u)} d u\right] \\
&\left.\times \int_{t}^{t_{k+1}} \frac{q(s)}{r(s)} \exp \left[\int_{t_{k+1}}^{s} \frac{r^{\prime}(u)+p(u)}{r(u)} d u\right)\right] d s .
\end{aligned}
$$

This contradicts the hypothesis.

\section{Remark}

When $\mathrm{r}(\mathrm{t})=1, \mathrm{p}(\mathrm{t})=0, \delta=0$, if we take $\mathrm{F}(\mathrm{t})=\mathrm{G}(\mathrm{t})=0$, the results of this paper become the ones of those given in $[4,5]$

\section{References}

[1] V.Lakshmikanthan,D.D.Bainov,P.Simeonov, Theory of Impulsive Differential equations, World Scientific,Singapore.

[2] Y.V.Rogovchenko,Oscillation theorem for second-order equations with damping,Nonliaear Anal. 41(2000)1005-1028.

[3] Xiaojing Yang, Oscillation criteria for nonlinear differential equations with damping,Applied Mathematics and Computation136(2003) 549-557.

[4] Y.Chen,W.Feng,oscillation theorem for second order non linear ODE equations with impulses,J.Math.Anal.Appl.210(1997)150-159.

[5] J.Luo,Z.Hou,Oscillation theorem for second order non linear ODE equation with impulses,J.Northeast Math.15 (1999)459-454.

[6] M.S.Peng,W.G.Ge,Oscillation criteria for second order nonlinear differential equations with impulses, Computers and Mathematics with Applications 39(2000)217-225.

[7] Wu Xiu-li, Chen Si -Yang ,Hong Ji ,Oscillation of a class of second order non linear ODE with impulses,Applied Mathematics and Computations 138 (2000)181-188.

[8] Xiaosong Tang,Asymptotic behaviour of solutions of second-order nonlinear delay differential equations with impulses,Journal of Computational and Applied Mathematics,233(2010)2105-2111 